\title{
Analysis and Study of Heat Transfer Resistance Inside and Outside the Reentry Capsule
}

\author{
Chen Yang, Ning Xianwen, Su Sheng \\ Beijing Institute of Spacecraft System Engineering, Beijing, 100094
}

\begin{abstract}
According to the heat transfer characteristics inside and outside of the lunar-earth high-speed reentry capsule, a typical calculation model of heat conduction in external thermal protection system(TPS) coupled with internal radiation was established. The thermal properties of thermal resistance inside and outside the reentry capsule were analysed. The effects of thickness of the TPS, surface conditions and atmospheric pressures on the temperature were further explored. The results showed that atmosphere pressure was necessary to be controlled under $10 \mathrm{~Pa}$ to ensure the safety temperature of the equipment and pipe. Based on the critical pressure, the configuration was optimized. The results provide detailed data for the system design of the lunar exploration, and also provide a reference for the thermal design of the atmospheric reentry spacecraft.
\end{abstract}

\section{Introduction}

In order to prevent the pneumatic heat eroding the inside wall in the return process, the outer surface of lunar-earth high-speed reentry capsule (reentry capsule) was fully coated with thermal protection system(TPS) ${ }^{1}$. So the enclosure formed a closed chamber, and the internal and external gas environment was unsmooth, and stated in a medium vacuum environment for long time.

The maximum heat insulation design ${ }^{2}$ is used inside and outside the reentry capsule. Figure 1(a) shows the overall shape of the reentry capsule. The outer mounted TPS is different from the recoverable satellite ${ }^{3}$ manned

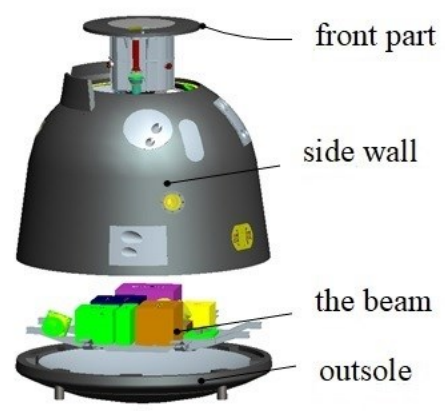

(a).overall shape of reentry capsule return module ${ }^{4}$, the reentry capsule is thicker and the thickness range is $28 \sim 66.5 \mathrm{~mm}$. The thermal resistance of the thermal protection has a great influence on the thermal resistance of the whole re-entry capsule. The metal side wall and beam are covered by multilayer heat insulation (MLI) inside to ensure the small heat leakage in the in-orbit storage stage and to restrain the high enthapy during the return process. The external and internal thermal resistance affect each other. Therefore, it is important to analyze the influence of thermal resistance of each layer on the temperature level of the whole reentry capsule.

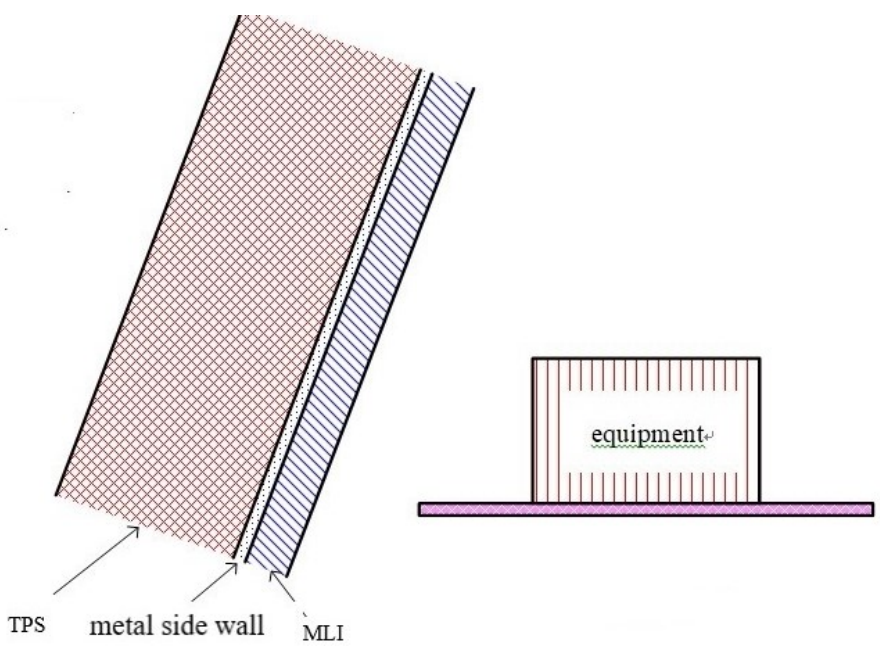

(b).physical model

Figure 1. Configuration of reentry capsule. 


\section{Reentry Capsule Heat Transfer Model}

\subsection{Physical Model}

Heat transfer resistance inside and outside the reentry capsule includes thermal resistance of TPS, thermal resistance of metal structure, multilayer radiation resistance and beam in medium vacuum environment, thermal resistance of equipment, etc. Figure 1(b) shows the installation of the side wall and the equipment. The TPS is installed on the aluminum plate. Inside of the aluminum plate is covered with MLI, the surface of the equipment is blackened, and the MLI and equipment are radiative heat transfer. The pipe diameter $\Phi 6 \mathrm{~mm}$, pipe clamps are made of non-metallic polyimide material and pipe mounting seat is aluminum base, welding with side wall.

\subsection{Mathematical Model}

The physical heat transfer process can be simplified as follows:

(1) The convection heat transfer can be neglected, due to the small space in the device.

(2) The influence of cable and cable support on equipment occlusion is not considered.

(3) The thickness of the metal side wall is only $0.5 \mathrm{~mm}$. It is considered that the temperatures of inner and outer layer of the MLI are equal.

Based on the above analysis, mathematical model is established as follows,

1) Temperature outside the thermal insulation structure

$m_{f} C_{p f} \frac{d T_{f}}{d \tau}=Q_{f}+K_{a f} A_{h}\left(\mathrm{~T}_{a}-\mathrm{T}_{f}\right)-\varepsilon_{f} \sigma A_{h} T_{f}^{4}$

2) Multilayer temperature

$m_{a} C_{p a} \frac{d T_{a}}{d \tau}=K_{a h} A_{h}\left(\mathrm{~T}_{h}-\mathrm{T}_{a}\right)-K_{a f} A_{h}\left(\mathrm{~T}_{a}-\mathrm{T}_{f}\right)$

3) Multi-layer inner temperature

$$
m_{h} C_{p h} \frac{d T_{h}}{d \tau}=K_{a h} A_{h}\left(\mathrm{~T}_{h}-\mathrm{T}_{a}\right)-\frac{\sigma A_{n r}}{1 / \varepsilon_{n}+A_{n}\left(1 / \varepsilon_{h}-1\right) A_{h}}\left(T_{n}^{4}-T_{h}^{4}\right)
$$

4) Equipment temperature

$m_{n} C_{p n} \frac{d T_{n}}{d \tau}=Q_{n}-Q_{W}-\frac{\sigma A_{n r}}{1 / \varepsilon_{n}+A_{n r}\left(1 / \varepsilon_{h}-1\right) A_{h}}\left(T_{n}^{4}-T_{h}^{4}\right)$

$m_{n}$ is quality of equipment, $m_{a}$ is MLI bulkhead side mass, $m_{h}$ is MLI inside side mass, $m_{f}$ is quality of the TPS, $Q_{f}$ is the absorbed external heat flux, $C_{p n}$ is specific heat capacity of equipment, $C_{p h}$ is MLI chamber film heat capacity, $C_{p a}$ is MLI bulkhead side heat capacity, $\mathrm{C}_{\mathrm{pf}}$ is heat capacity of TPS, $\mathrm{A}_{\mathrm{nw}}$ is contact area between equipment and mounting plate, $A_{h}$ is MLI area, $A_{f}$ is the TPS area, $\varepsilon_{n}$ is surface emissivity of equipment, $\varepsilon_{\mathrm{h}}$ is MLI emissivity, $\varepsilon_{\mathrm{f}}$ is TPS emissivity, $K_{a h}$ is MLI heat transfer coefficient, $K_{a f}$ is internal and external heat transfer coefficient of the TPS.

\section{Analysis of the Thermal Characteristics of the Reentry Capsule}

\subsection{Analytical Model Parameters and Boundary Conditions}

According to the characteristics of the overall configuration of the reentry capsule, the structural design and the system parameters, the analytical model parameters are determined, as shown in table 1.In the analysis, the cruising section with the worst low temperature of the reentry capsule is selected for analysis. The material parameters are taken at the beginning of the life, and the external heat flux is applied to the ship. The laser IMU and the typical pipe is selected for analysis and research.

Table 1. Analysis of model parameter values.

\begin{tabular}{cccccc}
\hline Code & Values & Code & Values & Code & Values \\
\hline $\mathrm{m}_{\mathrm{n}}$ & $7.65 \mathrm{~kg}$ (laser) & $\mathrm{A}_{\mathrm{nr}}$ & $0.185 \mathrm{~m}^{2}$ & $\mathrm{Q}_{\mathrm{f}}$ & $128 \mathrm{~W}$ \\
$\mathrm{~m}_{\mathrm{a}(\mathrm{h})}$ & $50.4 \mathrm{~g}$ & $\mathrm{~A}_{\mathrm{nw}}$ & $0.042 \mathrm{~m}^{2}$ & $\mathrm{Q}_{\mathrm{n}}$ & $16 \mathrm{~W}$ \\
$\mathrm{~m}_{\mathrm{f}}$ & $25.2 \mathrm{Kg}$ & $\mathrm{A}_{\mathrm{h}}$ & $5.04 \mathrm{~m}^{2}$ & $\mathrm{C}_{\mathrm{pn}}$ & $835 \mathrm{~J} / \mathrm{Kg} \cdot \mathrm{K}$ \\
$\mathrm{m}_{\mathrm{w}}$ & $0.93 \mathrm{~kg}$ & $\mathrm{~A}_{\mathrm{f}}$ & $5.04 \mathrm{~m}^{2}$ & $\mathrm{C}_{\mathrm{ph}}$ & $600 \mathrm{~J} / \mathrm{Kg} \cdot \mathrm{K}$ \\
$\mathrm{K}_{\mathrm{af}}$ & $0.00524 \mathrm{~W} / \mathrm{m}^{2} . \mathrm{K}$ & $\mathrm{A}_{\mathrm{w}}$ & $0.15 \mathrm{~m}^{2}$ & $\mathrm{C}_{\mathrm{pa}}$ & $600 \mathrm{~J} / \mathrm{Kg} \cdot \mathrm{K}$ \\
$\varepsilon_{\mathrm{w}}$ & 0.1 & $\varepsilon_{\mathrm{n}}$ & 0.88 & $\mathrm{C}_{\mathrm{pf}}$ & $1.4 \times 10^{-3} \mathrm{~J} / \mathrm{kg} \cdot \mathrm{K}$ \\
$\varepsilon_{\mathrm{f}}$ & 0.87 & $\varepsilon_{\mathrm{h}}$ & 0.1 & $\mathrm{C}_{\mathrm{pw}}$ & $900 \mathrm{~J} / \mathrm{Kg} \cdot \mathrm{K}$ \\
\hline
\end{tabular}




\subsection{Results and Analysis of Influencing Factors}

All painted white paint on the outside of the reentry capsule, the required heat of the propulsion system pipe is 16W2.In cruise attitude flight, only the top surface is radiated by external heat flux, and the absorption of external heat flux is relatively small.During cruise flight, the two IMU should meet the requirements of starting temperature $0^{\circ} \mathrm{C}$, and the pipe temperature(Tpipe) should be maintained above $5^{\circ} \mathrm{C}$. The steady-state results are as follows: the temperature of IMU (TIMU ) is $2.9{ }^{\circ} \mathrm{C}$, and the temperature of typical pipe is $5.9{ }^{\circ} \mathrm{C}$.

In the thermal resistance inside and outside the device, the MLI thermal resistance and the thermal resistance of the TPS are the two most important thermal resistances. The decrease of true test of MLI environment has a great influence on MLI performance56. The thermal resistance of TPS mainly depends on the thickness of the TPS. Therefore, the influence of MLI vacuum degree and thermal insulation thickness on the temperature of electronic equipment and pipe is evaluated by setting up the analysis condition of the influence of the vacuum degree and the thickness of the TPS, respectively.

Influence of thickness of the TPS

Under other conditions, the thickness of the TPS is reduced to $3 \sim 16 \mathrm{~mm}$ from the original $33 \mathrm{~mm}$, and the results are shown in table 2 . It can be seen that when the thickness of the TPS is reduced to $11 \mathrm{~mm}$, the temperature of the laser IMU will be less than $0^{\circ} \mathrm{C}$.

Table 2. Thickness of TPS.

\begin{tabular}{cccc}
\hline Working condition & Thickness $/ \mathrm{mm}$ & $\mathrm{T}_{\text {IMU }} /{ }^{\circ} \mathrm{C}$ & $\mathrm{T}_{\text {pipe }} /{ }^{\circ} \mathrm{C}$ \\
\hline Case 1 & 0.033 & 2.9 & 5.9 \\
Case 2 & 0.016 & 0.7 & 5.8 \\
Case 3 & 0.011 & -0.1 & 5.6 \\
Case 4 & 0.0055 & -0.4 & 5.2 \\
Case 5 & 0.0003 & -1.2 & 4.6 \\
\hline
\end{tabular}

(1) Vacuum effect

$\mathrm{T}_{\mathrm{IMU}}$ and $\mathrm{T}_{\text {pipe }}$ obtained by steady-state calculation under different vacuum conditions are shown in table 3 . With the pressure increasing, $\mathrm{T}_{\mathrm{IMU}}$ and $\mathrm{T}_{\text {pipe }}$ is reduced. When the pressure is greater than $10 \mathrm{~Pa}, \mathrm{~T}_{\text {pipe }}$ is lower than $5^{\circ} \mathrm{C}$.
When the pressure is more than $50 \mathrm{~Pa}, \mathrm{~T}_{\mathrm{IMU}}$ is lower than $0^{\circ} \mathrm{C}$. The temperature sensitivity of propulsion pipeline to vacuum degree is stronger than that of equipment because of the influence of multiple layers of bulk coating and multi-layer of side wall.

Table 3. Different vacuum analysis conditions.

\begin{tabular}{cccc}
\hline Working condition & Internal pressure $/ \mathrm{Pa}$ & $\mathrm{T}_{\mathrm{IMU}} /{ }^{\circ} \mathrm{C}$ & $\mathrm{T}_{\text {pipe }} /{ }^{\circ} \mathrm{C}$ \\
\hline Case 6 & 0.001 & 15.3 & 9.4 \\
Case 7 & 0.01 & 9.1 & 7 \\
Case 8 & 1 & 1 & 6.4 \\
Case 9 & 10 & 2.9 & 5.9 \\
Case 10 & 50 & 0.6 & 4.4 \\
Case 11 & 100 & -2.6 & 3.2 \\
\hline
\end{tabular}

(2) Effect of MLI surface condition

The influence of infrared emissivity is considered in the MLI of the device, and the polyester film, polyimide film and F46 face film are set up respectively.

It can be seen from table 4 that the heat transfer between the equipment and the bulkhead increases and the equipment temperature decreases with the increase of the MLI emissivity in the device.The reentry device is in the cruise section and the reentry section is designed for heat insulation. During the two flights, the heat control power is less. Therefore, the selection of polyester film on the multi-layer surface is the most favorable. 
Table 4. MLI surface condition.

\begin{tabular}{ccc}
\hline Working condition & MLI surface shape & $\mathrm{T}_{\mathrm{IMU}} /{ }^{\circ} \mathrm{C}$ \\
\hline Case 12 & Polyester film, emissivity 0.12 & 2.9 \\
Case 13 & F46 surface, emissivity0.68 & -13.9 \\
Case 14 & Polyimide film, emissivity0.88 & -15.8 \\
\hline
\end{tabular}

\section{Structural Optimization}

According to the results in Table 3 , if $\mathrm{T}_{\text {pipe }}$ can be controlled above $5^{\circ} \mathrm{C}$, the internal pressure value of the device cannot be greater than $10 \mathrm{~Pa}$.Since the pressure inside and outside of the return device is not smooth, it is necessary to design a suitable pressure relief hole without affecting the pneumatic, ablation and measurement and control, and to combine the idea of unified design of heat prevention and heat control grooming ${ }^{78}$. Therefore, two $\Phi$ $6 \mathrm{~mm}$ holes are designed at the front.The designed structure verifies the pressure relief ability of the through hole in the vacuum tank. After 7 hours of vacuum pumping, the pressure in the device decreases to 7.1 $\mathrm{Pa}$. In the heat balance test of the reentry capsule, two vacuum meters were installed at the inner side wall of the injector, and the vacuum degree in the device was monitored. The pressure reduced to $8.9 \mathrm{~Pa}$ in 6 hours. During the thermal test, the vacuum degree inside the device was higher than $0.12 \mathrm{~Pa}$.

\section{Conclusion}

Based on equilibrium thermal mathematical model, the matching between thermal resistances was analyzed. The pressure relief of different vacuum degree was investigated by numerical simulation. The influence of different thickness of the TPS and different surface condition on the temperature level in the device can be concluded as follows.(1)The effect of vacuum degree on the MLI of the reentry capsule was more serious. If the vacuum degree was greater than $10 \mathrm{~Pa}, \mathrm{~T}_{\text {pipe }}$ would not meet the requirements of the index. (2)The thermal resistance of the TPS was large, when the average thickness of TPS was reduced from $33 \mathrm{~mm}$ to $11 \mathrm{~mm}, \mathrm{~T}_{\text {IMU }}$ would be lower than $0{ }^{\circ} \mathrm{C}$, which can not meet the starting temperature index.(3)The MLI surface condition has a great influence on the temperature of the device. If the F46 or polyimide film was chosen, the temperature of the device would drop sharply, which can not meet the temperature requirement. Therefore, polyester film is beneficial to the insulation of cruise section and reentry section.

\section{Reference}

1. Yang M F, Zhang G, Zhang W, et al. Sci Sin Tech, 2015, 45:111-123.

2. Ning X W, Su S, Chen Y, et al. Sci Sin Tech, 2015, 45:145-150.

3. Dong $\mathrm{Y} Z$ Z. China Association of Science and Technology Annual Conference, Beijing, 2006.

4. Huang J R. Manned Spaceflight. 2012.2.

5. Chen Y, Ning X W, Su S, et al. Sci Sin Tech, 2015, 45: 263-267.

6. Liu W, Wen Y P. National Fifth Conference on Space Thermal Physics Huangshan, 2000

7. Wu G T. Spacecraft Engineering, 2009, 18(4):13-19.

8. Li W J, Liu F, Dong Y Z, Li X L. Spacecraft Engineering, 2013, 22(3):113-118. 